\title{
Ultranarrow Optical Inhomogeneous Linewidth in a Stoichiometric Rare-Earth Crystal
}

\author{
R. L. Ahlefeldt, ${ }^{1,2}$ M. R. Hush, ${ }^{3}$ and M. J. Sellars ${ }^{4}$ \\ ${ }^{1}$ Department of Physics, Montana State University, Bozeman, Montana 59717, USA \\ ${ }^{2}$ Laser Physics Centre, Research School of Physics and Engineering, The Australian National University, Canberra 0200, Australia \\ ${ }^{3}$ School of Engineering and Information Technology, \\ University of New South Wales at the Australian Defence Force Academy, Canberra 2600, Australia \\ ${ }^{4}$ Centre for Quantum Computation and Communication Technology, Research School of Physics and Engineering, \\ The Australian National University, Canberra 0200, Australia \\ (Received 19 January 2016; revised manuscript received 21 September 2016; published 15 December 2016)
}

\begin{abstract}
We obtain a low optical inhomogeneous linewidth of $25 \mathrm{MHz}$ in the stoichiometric rare-earth crystal $\mathrm{EuCl}_{3} \cdot 6 \mathrm{H}_{2} \mathrm{O}$ by isotopically purifying the crystal in ${ }^{35} \mathrm{Cl}$. With this linewidth, an important limit for stoichiometric rare-earth crystals is surpassed: the hyperfine structure of ${ }^{153} \mathrm{Eu}$ is spectrally resolved, allowing the whole population of ${ }^{153} \mathrm{Eu}^{3+}$ ions to be prepared in the same hyperfine state using hole-burning techniques. This material also has a very high optical density, and can have long coherence times when deuterated. This combination of properties offers new prospects for quantum information applications. We consider two of these: quantum memories and quantum many-body studies. We detail the improvements in the performance of current memory protocols possible in these high optical depth crystals, and describe how certain memory protocols, such as off-resonant Raman memories, can be implemented for the first time in a solid-state system. We explain how the strong excitation-induced interactions observed in this material resemble those seen in Rydberg systems, and describe how these interactions can lead to quantum many-body states that could be observed using standard optical spectroscopy techniques.
\end{abstract}

DOI: 10.1103/PhysRevLett.117.250504

The hyperfine levels of rare-earth ions have exceptionally long coherence times, as much as six hours in ${ }^{151} \mathrm{Eu}^{3+}[1]$. Additionally, these hyperfine levels are addressable through an intermediary optical level, allowing high-fidelity spin storage and read-out using optical pulses [2,3]. Because of these properties, doped rare-earth crystals have received considerable attention for quantum memory applications [2-6].

All the spin-wave quantum memory demonstrations to date have used rare-earth-doped crystals with low concentrations, ranging from $0.001 \%$ to $0.05 \%$ [2-4,7], resulting in low optical densities of the order of 1 to $10 \mathrm{~cm}^{-1}$. In addition, partly as a consequence of the disorder introduced by the rare-earth dopant, these crystals exhibited inhomogeneous linewidths of the order of $1 \mathrm{GHz}$, much greater than the hyperfine structure and the available Rabi frequencies. As a result, spectral-hole-burning techniques were necessary to create the narrow spectral features that would allow memory demonstrations. The use of these techniques, combined with the low initial optical density of the crystal, severely reduces the number of ions that can contribute to the operation of the memory (the usable optical depth), limiting its efficiency and storage density [8-10]. Because of these limitations, only two quantum memory demonstrations to date have surpassed the nocloning limit of 50\%, and in both cases a very large interaction length was required to obtain a sufficient optical depth to store even a single mode [4,11].

Narrow-linewidth high-optical-density materials would therefore be very beneficial for quantum memory applications. Very narrow inhomogeneous linewidths have been observed in rare-earth-doped crystals by reducing the dopant concentration to the ppm level and by isotopically purifying the host. The most well-known example is $\mathrm{YLiF}_{4}$ [12-14]. When isotopically purified in ${ }^{7} \mathrm{Li}$ to remove broadening caused by isotopic disorder, this material has optical inhomogeneous linewidths as low as $16 \mathrm{MHz}$ for $\mathrm{Er}^{3+}$ [15] and $10 \mathrm{MHz}$ for $\mathrm{Nd}^{3+}$ [16]. To achieve such narrow lines, it was necessary to use extremely low rareearth dopant concentrations, around $3 \mathrm{ppm}$. So, although these crystals have much lower disorder than current memory materials, their optical and spatial densities are low, and little advantage is gained from their narrow lines for quantum memory purposes.

In this Letter, we show that small inhomogeneous linewidths can be achieved at the same time as high optical and spatial densities by isotopically purifying a material that is stoichiometric, rather than doped, in the rare-earth ion. The linewidth reduction is sufficient in our chosen material, $\mathrm{EuCl}_{3} \cdot 6 \mathrm{H}_{2} \mathrm{O}$, that the rare-earth hyperfine structure is resolved. Reaching this limit in the high optical depth regime has broad-reaching implications for quantum memories. It also opens new applications, and in particular we discuss the use of these materials for many-body physics studies, which are made possible because the linewidth is also smaller than the nearest-neighbor ionion interactions [17].

$\mathrm{EuCl}_{3} \cdot 6 \mathrm{H}_{2} \mathrm{O}$ is a good starting point in the quest to achieve ultranarrow linewidths because it already has a very 
narrow, $100 \mathrm{MHz}$ optical linewidth without isotopic purification [18], and good coherence times when deuterated [19]. This makes it an excellent quantum memory candidate in its own right.

$\mathrm{EuCl}_{3} \cdot 6 \mathrm{H}_{2} \mathrm{O}$ is a monoclinic crystal with a single rareearth site of $C_{2}$ site symmetry [20,21]. The environment of a Eu${ }^{3+}$ ion in $\mathrm{EuCl}_{3} \cdot 6 \mathrm{H}_{2} \mathrm{O}$ is shown in Fig. 1. It has eight direct ligands-six water molecules and two $\mathrm{Cl}$ ions at distances between 2.4 and $2.8 \AA$. Also shown are the first shell of $\mathrm{Eu}^{3+}$ neighbors.

The optical ${ }^{7} F_{0} \rightarrow{ }^{5} D_{0}$ transition of $\mathrm{EuCl}_{3} \cdot 6 \mathrm{H}_{2} \mathrm{O}$ at $579.703 \mathrm{~nm}$ (vacuum) shows a structured line spread over $600 \mathrm{MHz}$, with each component line approximately $100 \mathrm{MHz}$ wide. The structure of the line can be completely explained by a combination of the hyperfine structure of the two europium isotopes and shifts caused by different isotopes occupying the nearest-neighbor ligand positions [18]. These isotope shifts can be large, up to $2 \mathrm{GHz}$ for $\mathrm{Eu}^{3+}$ ions neighboring $\mathrm{D}$ ions $\left({ }^{2} \mathrm{H}\right)$, and we expect that isotopes occupying more distant sites contribute substantially to the inhomogeneous broadening.

The contribution of the different isotopes to the broadening can be estimated simply. It depends on the concentration of that element in the crystal, the abundance of the isotope relative to the dominant isotope, and the perturbation to the lattice caused by substituting the isotope. This latter quantity depends on the relative mass difference of the isotope and the dominant isotope, thus D causes the largest perturbation and ${ }^{151} \mathrm{Eu}^{3+}$ the smallest.

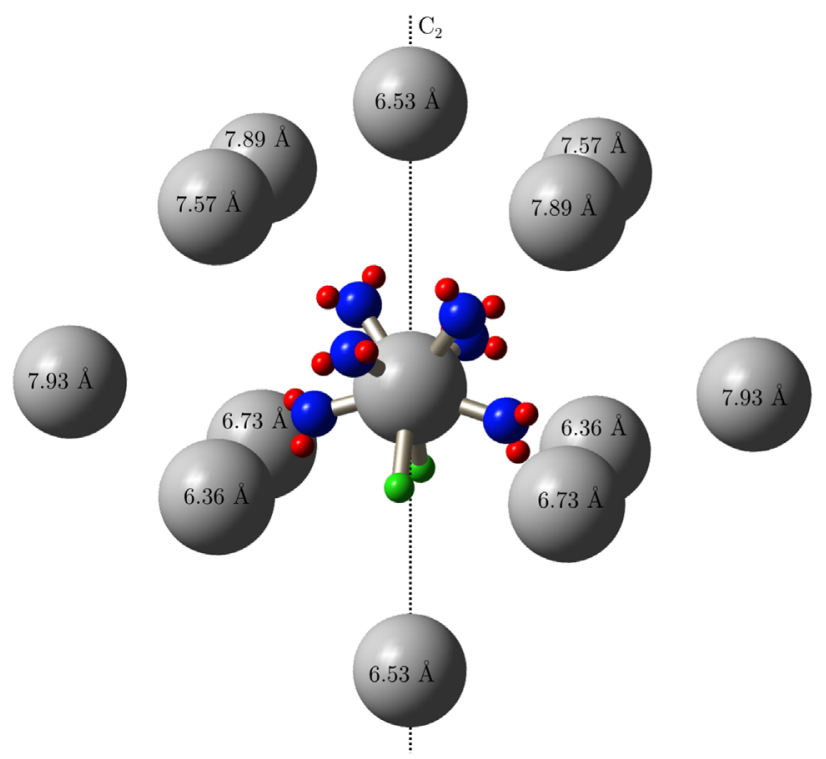

FIG. 1. The first shell of $\mathrm{Eu}^{3+}$ ions (gray) in $\mathrm{EuCl}_{3} \cdot 6 \mathrm{H}_{2} \mathrm{O}$. The $C_{2}$ symmetry axis is vertical. The $\mathrm{Eu}^{3+}$ ions are labeled by their distance from the central ion. Ligand ions $\mathrm{Cl}$ (green), $\mathrm{O}$ (blue), and $\mathrm{H}$ (red) are only shown for the central ion. Our previous measurements suggest that the static interaction between ions separated by $6.53 \AA$ along the $C_{2}$ axis is $>40 \mathrm{MHz}$.
We have measured the inhomogeneous broadening rate due to $\mathrm{D}$ as $91 \mathrm{MHz} \%$ concentration [18], suggesting that $\mathrm{D}$ is responsible for $1.4 \mathrm{MHz}$ of the inhomogeneous broadening seen in natural abundance crystals. Assuming the broadening scales linearly with the relative mass difference, the concentration, and the abundance, we expect that ${ }^{17} \mathrm{O}$ and ${ }^{18} \mathrm{O}$ contribute $\approx 2 \mathrm{MHz}$ to the inhomogeneous broadening, ${ }^{37} \mathrm{Cl} \approx 60 \mathrm{MHz}$, and ${ }^{151} \mathrm{Eu}^{3+} \approx 10 \mathrm{MHz}$. These numbers are approximate, but indicate that ${ }^{37} \mathrm{Cl}$ will be the major source of broadening in $\mathrm{EuCl}_{3} \cdot 6 \mathrm{H}_{2} \mathrm{O}$.

To reduce the crystal linewidth, we grew a crystal isotopically purified in ${ }^{35} \mathrm{Cl}$ from a water solution at near room temperature. The starting material was $6 \mathrm{~g}$ of $\mathrm{Eu}^{35} \mathrm{Cl}_{3} \cdot 6 \mathrm{H}_{2} \mathrm{O}$ that had been prepared from $\mathrm{Na}^{35} \mathrm{Cl}$ and $99.999 \% \mathrm{EuCl}_{3} \cdot 6 \mathrm{H}_{2} \mathrm{O}$. The nominal isotopic purity was $99.67 \%$. Sufficient water was added to saturate the solution at $30^{\circ} \mathrm{C}$. A crystal was grown out of the solution over one week after lowering the temperature to $29^{\circ} \mathrm{C}$. The growth rate during this time was not constant as the hotplate used for growth only has a temperature stability of $\pm 1{ }^{\circ} \mathrm{C}$. An unstable growth rate can lead to inclusions and other growth defects. Because the base linewidth in this material is so low, the effect of these rare growth defects can be seen as small macroscopic variations in the inhomogeneous linewidth of the order of $20 \mathrm{MHz}$ over millimeter-size regions of the crystal. We expect that the majority of these defects and the associated macroscopic variations in broadening can be removed by improving the temperature stability of the growth process.

The excitation spectrum of the ${ }^{7} F_{0} \rightarrow{ }^{5} D_{0}$ transition was measured with a Coherent 699-29 ring dye laser with the crystal at $4 \mathrm{~K}$ in a helium bath cryostat. Because $\mathrm{EuCl}_{3}$. $6 \mathrm{H}_{2} \mathrm{O}$ is hygroscopic, the crystal was mounted in a small helium-gas-filled chamber to avoid exposure to air or vacuum. The laser beam was orientated parallel to the $C_{2}$ axis to minimize absorption. Even along this direction, the absorption is high, and a confocal imaging system was used to collect fluorescence only from the front face of the crystal. In this setup, achromatic $10 \mathrm{~cm}$ lenses were used for objective and imaging lenses, and the emission was focused onto the end of a $62.5 \mu \mathrm{m}$ multimode fiber serving as the pinhole.

Figure 2 shows the excitation spectrum of $\mathrm{Eu}^{35} \mathrm{Cl}_{3} \cdot 6 \mathrm{H}_{2} \mathrm{O}$. There is a $200 \mathrm{MHz} \mathrm{Eu}{ }^{3+}$ isotope shift in $\mathrm{EuCl}_{3} \cdot 6 \mathrm{H}_{2} \mathrm{O}$, leading to two sets of peaks: around the $0 \mathrm{MHz}$ frequency offset are those due to ${ }^{151} \mathrm{Eu}^{3+}$, while at higher frequencies are those due to ${ }^{153} \mathrm{Eu}^{3+}$. These sets of peaks are split by the hyperfine interaction. For ${ }^{153} \mathrm{Eu}^{3+}$, which has the larger hyperfine splittings, this hyperfine structure is well resolved. In Fig. 2, we have labeled each peak for ${ }^{153} \mathrm{Eu}^{3+}$ with the transition that generates it.

The inhomogeneous linewidth of each line in the spectrum is $25 \pm 5 \mathrm{MHz}$. The instrumental broadening due to instability in the laser scan is less than $5 \mathrm{MHz}$. 


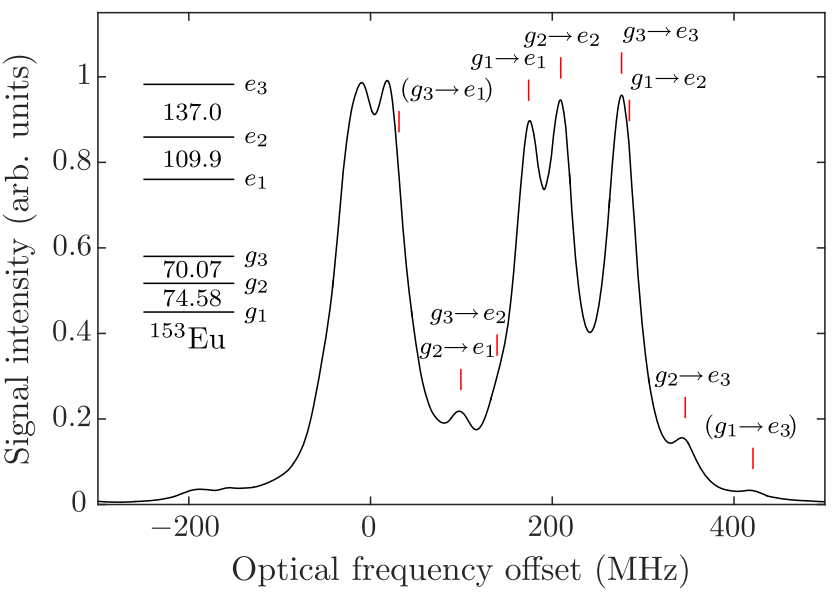

FIG. 2. Excitation spectrum of the ${ }^{7} F_{0} \rightarrow{ }^{5} D_{0}$ transition in isotopically pure $\mathrm{Eu}^{35} \mathrm{Cl}_{3} \cdot 6 \mathrm{H}_{2} \mathrm{O}$. The energy level structure of the ${ }^{7} F_{0}$ ground and ${ }^{5} D_{0}$ excited states for ${ }^{153} \mathrm{Eu}^{3+}$ is shown on the upper left, where the splittings are given in megahertz. The positions of each transition between the ${ }^{153} \mathrm{Eu}^{3+}$ ground and excited states are indicated by red lines on the spectrum. The weak transitions are bracketed.

As described earlier, we expect that about $10 \mathrm{MHz}$ of the remaining linewidth is due to ${ }^{151} \mathrm{Eu}^{3+}$, with about $3 \mathrm{MHz}$ due to the extra $\mathrm{O}$ and $\mathrm{H}$ isotopes. Therefore, isotopic purification of the other elements in the crystal will reduce the linewidth to the order of $10 \mathrm{MHz}$.

We also studied spectral hole burning in the crystal using the same setup. We burned holes with $12 \mathrm{~mW}$ of laser power over $4.5 \mathrm{~s}$, then swept $400 \mathrm{MHz}$ over the ${ }^{153} \mathrm{Eu}^{3+}$ peaks with lower power, $2.5 \mathrm{~mW}$, to minimize alteration of the hole-burning spectrum by the read-out sweep. We chose to burn holes on the $g_{2} \rightarrow e_{3}$ peak of ${ }^{153} \mathrm{Eu}$. This preferentially pumps population into $g_{3}$ because most of the oscillator strength from the $e_{3}$ state goes into the $e_{3} \rightarrow g_{3}$ transition. The resulting hole had a linewidth of $4 \mathrm{MHz}$, consistent with the long term linewidth of the laser.

Figure 3 shows the structure associated with this hole (burned at $340 \mathrm{MHz}$, peak $A$ ). As well as the expected side and antiholes, extra features are seen. For example, as well as the expected antihole separated from the hole by the $g_{2}-g_{3}$ splitting (peak $C$ ), there is another, broader antihole centered exactly on the $g_{2} \rightarrow e_{3}$ line (peak $B$ ). This feature and the associated broad side holes seen on the $g_{1} \rightarrow e_{1}$ and $g_{2} \rightarrow e_{2}$ transitions are created by hyperfine spin-spin cross relaxation of hole burned ions with ions nonresonant with the laser during the hole-burning step. This results in extra optical pumping of ions from both $g_{1}$ and $g_{2}$ into $g_{3}$. Despite the cross relaxation and the relatively weak excitation, the original hole is very deep, $70 \%$.

The cross relaxation occurs because the hyperfine inhomogeneous broadening is small and the $\mathrm{Eu}^{3+}$ ions are close together, allowing mutual spin flips to occur. The lifetime of the original hole is of the order of $10 \mathrm{~s}$, giving a

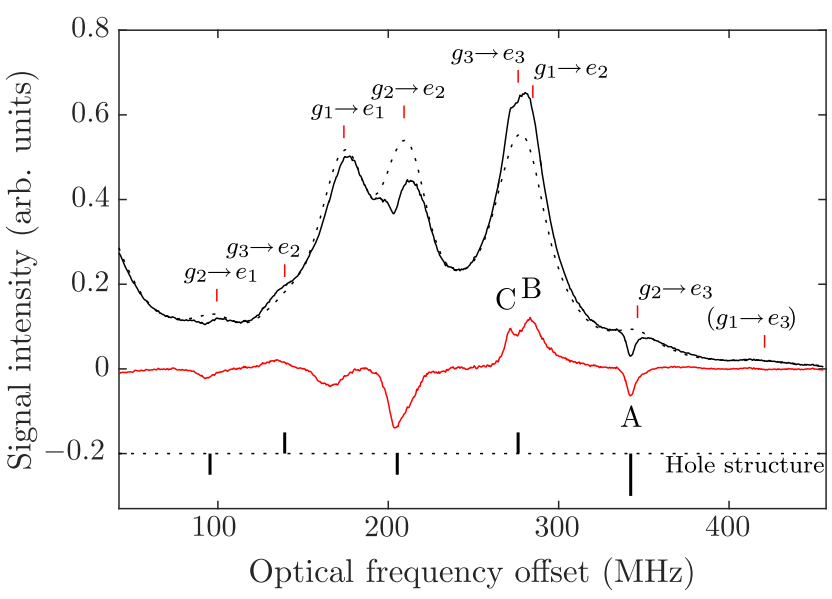

FIG. 3. Hole-burning spectrum in $\mathrm{Eu}^{35} \mathrm{Cl}_{3} \cdot 6 \mathrm{H}_{2} \mathrm{O}$ (solid black line) with an unburned spectrum for reference (dotted line). The red trace in the center is the difference of these two spectra, and the expected hole or antihole structure due to transferring population from $g_{2}$ to $g_{3}$ is indicated at the bottom of the figure. Three peaks are labeled in the figure. Peak $A$ is the original hole, peak $C$ is a direct antihole, and peak $B$ is a second antihole resulting from cross relaxation (see text).

time scale for this cross-relaxation process. However, the cross-relaxed side and antiholes persist for much longer, of the order of $1 \mathrm{~h}$. This is comparable to the spin-lattice relaxation rate of $5 \mathrm{~h}$ that we see in $\mathrm{EuCl}_{3} \cdot 6 \mathrm{H}_{2} \mathrm{O}$, indicating that there is no significant spatial diffusion of the excitation on this time scale.

The results above demonstrate that $\mathrm{Eu}^{35} \mathrm{Cl}_{3} \cdot 6 \mathrm{H}_{2} \mathrm{O}$ has three very useful features for quantum information applications: a very high optical density, an inhomogeneous linewidth smaller than the hyperfine structure and nearestneighbor interactions [17], and the ability to burn deep spectral features with a good lifetime, even in the presence of cross relaxation. In addition, very long coherence times will be possible by deuterating the crystal to improve the optical coherence time to the order of $1 \mathrm{~ms}$ [19], and by using the zero-first-order Zeeman technique [22] to extend the hyperfine coherence time towards the lifetime limit. Below, we discuss the implications of these properties for two quantum information applications: quantum memories and many-body-physics studies. Other quantum information applications, such as optical delay lines [23] and DLCZ-type quantum repeaters [24,25], could also benefit.

We will first consider quantum memories, concentrating on spin-wave storage quantum memory protocols, which comprise the atomic frequency comb (AFC) [23], the gradient echo memory (GEM) [26,27], and Raman-type protocols such as electromagnetically induced transparency (EIT) $[28,29]$. These protocols make use of some type of optical pumping preparation-either for tailoring spectral features (AFC, GEM), or to prepare the material in a single spin ground state (most Raman schemes), so the ability to burn spectral holes in $\mathrm{Eu}^{35} \mathrm{Cl}_{3} \cdot 6 \mathrm{H}_{2} \mathrm{O}$ shown above is crucial. 
The cross relaxation seen here may affect the ability to produce very sharp spectral features; however, a simple solution to turn off the cross relaxation is to reduce the ${ }^{153} \mathrm{Eu}^{3+}$ concentration to below $10 \%$ using isotope doping. This has the added advantage of reducing further the inhomogeneous broadening, maintaining a high optical density.

Based on the transition oscillator strength $\left(3 \times 10^{-9}\right)$ and $\mathrm{Eu}^{3+}$ ion concentration, current $\mathrm{Eu}^{35} \mathrm{Cl}_{3} \cdot 6 \mathrm{H}_{2} \mathrm{O}$ crystals are likely to have an optical density of the order of $1000 \mathrm{~cm}^{-1}$. It is this extremely high optical density that is most beneficial for memories, whose performance to date has been restricted by the $\approx 1 \mathrm{~cm}^{-1}$ optical densities of current crystals. Multiple aspects of memory performance will be improved. First, for all protocols the efficiency, which is directly dependent on the optical depth, will be increased. In $\mathrm{Eu}^{35} \mathrm{Cl}_{3} \cdot 6 \mathrm{H}_{2} \mathrm{O}$, a memory efficiency above $90 \%$ should be easily achievable, exceeding the highest memory efficiency seen, $87 \%$ in $\mathrm{Rb}$ gas [30].

Second, the multimode storage capacity will also be increased across all protocols; this is ultimately dependent on how many atoms are available to store different modes, and therefore on the optical depth. For example, if spatial multiplexing is used, since $\mathrm{Eu}^{35} \mathrm{Cl}_{3} \cdot 6 \mathrm{H}_{2} \mathrm{O}$ will show appreciable absorption over $1 \mu \mathrm{m}$, it will be possible to create a dense set of spatial voxels each capable of storing a mode at high efficiency.

Finally, protocol-dependent improvements are also possible. For example, while the GEM and EIT protocols allow higher initial efficiency than the AFC protocol, the bandwidth is dependent on the optical depth, so there is eventually a trade-off between efficiency and bandwidth or spectral or temporal multiplexing capacity [9]. This trade-off is alleviated by a very high optical depth, allowing large-bandwidth high-efficiency storage. For the GEM protocol, a high optical density should allow the bandwidth limit, the ground state hyperfine splitting, to be reached. This limit is generally considered to apply to all spectral hole burning-based memories like the GEM and AFC protocols. However, for a material like $\mathrm{Eu}^{35} \mathrm{Cl}_{3} \cdot 6 \mathrm{H}_{2} \mathrm{O}$ in which the hyperfine structure is resolved, this limitation is lifted in the GEM protocol [4], allowing much larger memory bandwidths.

In addition to these performance improvements in current protocols, new memory protocols will be possible in $\mathrm{Eu}^{35} \mathrm{Cl}_{3} \cdot 6 \mathrm{H}_{2} \mathrm{O}$. In particular, the ability to resolve the hyperfine structure allows off-resonant Raman schemes for the first time in a rare-earth system [31,32]. These protocols require a high optical depth, and that the system be prepared into a single hyperfine ground state over the entire spectral width of the line. The spin-spin cross relaxation seen here provides a very efficient way of preparing this type of state in $\mathrm{Eu}^{35} \mathrm{Cl}_{3} \cdot 6 \mathrm{H}_{2} \mathrm{O}$. Off-resonant Raman protocols have a number of advantages over the resonant schemes described above, such as lower noise because the excited state is never populated.
Similar to Raman memories, the second quantum information application we will consider, quantum many-body dynamics, is enabled in $\mathrm{Eu}^{35} \mathrm{Cl}_{3} \cdot 6 \mathrm{H}_{2} \mathrm{O}$ by the high optical density and small optical inhomogeneous broadening. In this material, the $\mathrm{Eu}^{3+}$ atoms are close together and can have interactions much stronger than the residual inhomogeneity in the system. The result is a lattice of interacting $\mathrm{Eu}^{3+}$ atoms, the ideal platform for investigating many-body quantum phenomena.

This would involve preparing the ions in a single ground state as for Raman memories, and exciting the optical transition to observe many-body effects. The interactions on the optical transition will determine the many-body states seen. A variety of interaction mechanisms, such as electric and magnetic multipole and superexchange (wave-function overlap mediated by ligands), are possible, but all can be divided into two parts. The first is a diagonal interaction producing a static shift to the electronic levels $H_{d} / h=V(r)\left|e_{3}, e_{3}\right\rangle \times$ $\left\langle e_{3}, e_{3}\right|$. We previously measured these nearest-neighbor interactions to be on the order of $40 \mathrm{MHz}$ in $\mathrm{EuCl}_{3} \cdot 6 \mathrm{H}_{2} \mathrm{O}$ [17], larger than the inhomogeneous linewidth. The second interaction is an off-diagonal interaction, of the form $H_{o} / h=T(r)\left|e_{3}, g_{3}\right\rangle\left\langle g_{3}, e_{3}\left|+T^{*}(r)\right| g_{3}, e_{3}\right\rangle\left\langle e_{3}, g_{3}\right|$.

We expect the diagonal interaction to dominate in $\mathrm{Eu}^{35} \mathrm{Cl}_{3} \cdot 6 \mathrm{H}_{2} \mathrm{O}$ over all length scales. Over short ion separations, if the off-diagonal interaction were dominant, it would cause the inhomogeneous linewidth to be dependent on the ion density, which we do not see. In the long range, we can directly estimate the ratio of diagonal and off-diagonal interactions since we can assume the interactions are dipolar: it will be the ratio of the static $d_{\text {static }}=1.0 \times 10^{-32} \mathrm{C} \mathrm{m}$ and transition $d_{\text {trans }}=1.6 \times 10^{-33} \mathrm{C} \mathrm{m}$ dipole moments squared [17]. Thus, the diagonal interaction will be $d_{\text {static }}^{2} / d_{\text {trans }}^{2}=42$ times stronger than the off-diagonal interactions.

As the diagonal interaction dominates, a close analogue for $\mathrm{Eu}^{35} \mathrm{Cl}_{3} \cdot 6 \mathrm{H}_{2} \mathrm{O}$ among cold atom systems is an optical lattice of Rydberg atoms [33,34], and the many-body effects predicted for Rydberg systems should also be observable in our rare-earth ion setting. Currently, the primary experimental signature used to verify the presence of Rydberg interactions is the blockade effect [35,36]: exciting one atom in the system pushes nearby atoms out of resonance with the exciting field. Since the ion-ion distances and the associated blockade region are much smaller in solid-state systems, the spatial imaging methods used to study Rydberg systems are not applicable. Spectral imaging, or sub diffraction-limited spectral-spatial imaging [37], could be used instead. The blockade region could also be observed indirectly through the suppression of excitation [36] or EIT spectroscopy [38].

There are a variety of many-body states predicted to exist for Rydberg systems that could be investigated in rare-earth crystals [39-46]. In the dense excitation limit, rare-earth crystals may have an advantage over Rydberg systems: Rydberg lattices have recently shown atomic-density 
dependent broadening as a consequence of the two-photon transitions used to prepare the system [47], which is likely to bar them from the high density regime.

In conclusion, we have shown that $\mathrm{EuCl}_{3} \cdot 6 \mathrm{H}_{2} \mathrm{O}$ isotopically purified in ${ }^{35} \mathrm{Cl}$ has an optical inhomogeneous linewidth for the ${ }^{7} F_{0} \rightarrow{ }^{5} D_{0}$ transition of $\mathrm{Eu}^{3+}$ of $25 \mathrm{MHz}$, the lowest of any stoichiometric rare-earth crystal and one of the lowest inhomogeneous linewidths measured in any material. Isotopic purification of the other elements in the crystal, in particular $\mathrm{Eu}^{3+}$, will further lower this linewidth. Even in this very-high-density system, it is possible to spin polarize the sample, and to prepare spectrally narrow features. Combined with this, the small linewidth and high optical depth mean this material would be a good system for quantum memories. This material also shows strong excitation induced frequency shifts between nearest neighbors, which suggests that many-body physics analogous to that seen in Rydberg systems can be observed.

This work was supported by the Australian Research Council Centre of Excellence for Quantum Computation and Communication Technology (Grant No. CE110001027). M.R.H. acknowledges funding from an Australian Research Council (ARC) Discovery Project (Project No. DP140101779). M. J. S. was supported by an Australian Research Council Future Fellowship (Grant No. FT110100919). The authors thank E. A. Goldschmidt for helpful discussions.

[1] M. Zhong, M. P. Hedges, R. L. Ahlefeldt, J. G. Bartholomew, S. E. Beavan, S. M. Wittig, J. J. Longdell, and M. J. Sellars, Optically addressable nuclear spins in a solid with a six-hour coherence time, Nature (London) 517, 177 (2015).

[2] P. Jobez, C. Laplane, N. Timoney, N. Gisin, A. Ferrier, P. Goldner, and M. Afzelius, Coherent Spin Control at the Quantum Level in an Ensemble-Based Optical Memory, Phys. Rev. Lett. 114, 230502 (2015).

[3] M. Gündoğan, P. M. Ledingham, K. Kutluer, M. Mazzera, and H. de Riedmatten, Solid State Spin-Wave Quantum Memory for Time-Bin Qubits, Phys. Rev. Lett. 114, 230501 (2015).

[4] M. P. Hedges, J. J. Longdell, Y. Li, and M. J. Sellars, Efficient quantum memory for light, Nature (London) 465, 1052 (2010).

[5] J. Dajczgewand, J.-L. Le Gouët, A. Louchet-Chauvet, and T. Chanelière, Large efficiency at telecom wavelength for optical quantum memories, Opt. Lett. 39, 2711 (2014).

[6] E. Saglamyurek, J. Jin, V. B. Verma, M. D. Shaw, F. Marsili, S. W. Nam, D. Oblak, and W. Tittel, Quantum storage of entangled telecom-wavelength photons in an erbium-doped optical fibre, Nat. Photonics 9, 83 (2015).

[7] M. Afzelius, I. Usmani, A. Amari, B. Lauritzen, A. Walther, C. Simon, N. Sangouard, J. Minář, H. de Riedmatten, N. Gisin, and S. Kröll, Demonstration of Atomic Frequency Comb Memory for Light With Spin-Wave Storage, Phys. Rev. Lett. 104, 040503 (2010).
[8] A. V. Gorshkov, A. André, M. Fleischhauer, A. S. Sørensen, and M.D. Lukin, Universal Approach to Optimal Photon Storage in Atomic Media, Phys. Rev. Lett. 98, 123601 (2007).

[9] J. Nunn, K. Reim, K. C. Lee, V. O. Lorenz, B. J. Sussman, I. A. Walmsley, and D. Jaksch, Multimode Memories in Atomic Ensembles, Phys. Rev. Lett. 101, 260502 (2008).

[10] P. Jobez, N. Timoney, C. Laplane, J. Etesse, A. Ferrier, P. Goldner, N. Gisin, and M. Afzelius, Towards highly multimode optical quantum memory for quantum repeaters, Phys. Rev. A 93, 032327 (2016).

[11] D. Schraft, M. Hain, N. Lorenz, and T. Halfmann, Stopped light at high storage efficiency in a $\operatorname{Pr}^{3+}: \mathrm{Y}_{2} \mathrm{SiO}_{5}$ crystal, Phys. Rev. Lett. 116, 073602 (2016).

[12] N. I. Agladze, M. N. Popova, G. N. Zhizhin, V. J. Egorov, and M. A. Petrova, Isotope structure in optical spectra of $\mathrm{LiYF}_{4}: \mathrm{Ho}^{3+}$, Phys. Rev. Lett. 66, 477 (1991).

[13] R. M. Macfarlane, A. Cassanho, and R. S. Meltzer, Inhomogeneous Broadening by Nuclear Spin Fields: A New Limit for Optical Transitions in Solids, Phys. Rev. Lett. 69, 542 (1992).

[14] E. Chukalina, M. Popova, S. Korableva, and R. Abdulsabirov, Fine structure of spectral lines in $\mathrm{LiYF}_{4}: \mathrm{Er}^{3+}$ due to isotopic disorder in the lattice, Phys. Lett. A 269, 348 (2000).

[15] C. Thiel, T. Böttger, and R. Cone, Rare-earth-doped materials for applications in quantum information storage and signal processing, J. Lumin. 131, 353 (2011).

[16] R. M. Macfarlane, R. S. Meltzer, and B. Z. Malkin, Optical measurement of the isotope shifts and hyperfine and superhyperfine interactions of $\mathrm{Nd}$ in the solid state, Phys. Rev. B 58, 5692 (1998).

[17] R. L. Ahlefeldt, D. L. McAuslan, J. J. Longdell, N. B. Manson, and M.J. Sellars, Precision Measurement of Electronic Ion-Ion Interactions Between Neighboring $\mathrm{Eu}^{3+}$ Optical Centers, Phys. Rev. Lett. 111, 240501 (2013).

[18] R. L. Ahlefeldt, A. Smith, and M. J. Sellars, Ligand isotope structure of the optical ${ }^{7} F_{0} \rightarrow{ }^{5} D_{0}$ transition in $\mathrm{EuCl}_{3} \cdot 6 \mathrm{H}_{2} \mathrm{O}$, Phys. Rev. B 80, 205106 (2009).

[19] R. Ahlefeldt, N. Manson, and M. Sellars, Optical lifetime and linewidth studies of the ${ }^{7} F_{0} \rightarrow{ }^{5} D_{0}$ transition in $\mathrm{EuCl}_{3} \cdot 6 \mathrm{H}_{2} \mathrm{O}$ : A potential material for quantum memory applications, J. Lumin. 133, 152 (2013).

[20] N. K. Bel'skii and Y. T. Struchkov, The crystal structure and optical properties of europium chloride hexahydrate, $\mathrm{EuCl}_{3} \cdot 6 \mathrm{H}_{2} \mathrm{O}$, Sov. Phys. Crystallogr. 10, 15 (1965).

[21] D. L. Kepert, J. M. Patrick, and A. H. White, Structure and stereochemistry in 'f-block' complexes of high coordination number. VII. The $\left.\left[\mathrm{M}(\text { unindentate } \mathrm{X})_{2} \text { (unindentate } \mathrm{Y}\right)_{6}\right]$ system: Crystal structure of hexaaquadichloroeuropium (III) chloride (a redetermination), Australian Journal of Chemistry 36, 477 (1983).

[22] E. Fraval, M. J. Sellars, and J. J. Longdell, Method of Extending Hyperfine Coherence Times in $\operatorname{Pr}^{3+}: \mathrm{Y}_{2} \mathrm{SiO}_{5}$, Phys. Rev. Lett. 92, 077601 (2004).

[23] M. Afzelius, C. Simon, H. de Riedmatten, and N. Gisin, Multimode quantum memory based on atomic frequency combs, Phys. Rev. A 79, 052329 (2009).

[24] L.-M. Duan, M. D. Lukin, J. I. Cirac, and P. Zoller, Longdistance quantum communication with atomic ensembles and linear optics, Nature (London) 414, 413 (2001). 
[25] P. M. Ledingham, W. R. Naylor, J. J. Longdell, S. E. Beavan, and M. J. Sellars, Nonclassical photon streams using rephased amplified spontaneous emission, Phys. Rev. A 81, 012301 (2010).

[26] A. Alexander, J. Longdell, M. Sellars, and N. Manson, Coherent information storage with photon echoes produced by switching electric fields, J. Lumin. 127, 94 (2007).

[27] G. Hetet, J. J. Longdell, A. L. Alexander, P. K. Lam, and M. J. Sellars, Electro-Optic Quantum Memory for Light Using Two-Level Atoms, Phys. Rev. Lett. 100, 023601 (2008).

[28] M. Fleischhauer and M. D. Lukin, Quantum memory for photons: Dark-state polaritons, Phys. Rev. A 65, 022314 (2002).

[29] S. E. Harris, J. E. Field, and A. Imamoğlu, Nonlinear Optical Processes Using Electromagnetically Induced Transparency, Phys. Rev. Lett. 64, 1107 (1990).

[30] Y.-W. Cho, G. T. Campbell, J. L. Everett, J. Bernu, D. B. Higginbottom, M. T. Cao, J. Geng, N. P. Robins, P. K. Lam, and B.C. Buchler, Highly efficient optical quantum memory with long coherence time in cold atoms, Optica 3, 100 (2016).

[31] K. F. Reim, J. Nunn, V. O. Lorenz, B. J. Sussman, K. C. Lee, N. K. Langford, D. Jaksch, and I. A. Walmsley, Towards high-speed optical quantum memories, Nat. Photonics 4, 218 (2010).

[32] S. A. Moiseev, Off-resonant Raman-echo quantum memory for inhomogeneously broadened atoms in a cavity, Phys. Rev. A 88, 012304 (2013).

[33] S. E. Anderson, K. C. Younge, and G. Raithel, Trapping Rydberg Atoms in an Optical Lattice, Phys. Rev. Lett. 107, 263001 (2011).

[34] M. Viteau, M. G. Bason, J. Radogostowicz, N. Malossi, D. Ciampini, O. Morsch, and E. Arimondo, Rydberg Excitations in Bose-Einstein Condensates in Quasi-OneDimensional Potentials and Optical Lattices, Phys. Rev. Lett. 107, 060402 (2011).

[35] Y. O. Dudin and A. Kuzmich, Strongly interacting Rydberg excitations of a cold atomic gas, Science 336, 887 (2012).
[36] D. Tong, S. M. Farooqi, J. Stanojevic, S. Krishnan, Y. P. Zhang, R. Côté, E. E. Eyler, and P. L. Gould, Local Blockade of Rydberg Excitation in an Ultracold Gas, Phys. Rev. Lett. 93, 063001 (2004).

[37] S. Schiller and R. L. Byer, Subwavelength optical magneticresonance imaging, J. Opt. Soc. Am. A 9, 683 (1992).

[38] J. D. Pritchard, D. Maxwell, A. Gauguet, K. J. Weatherill, M. P. A. Jones, and C. S. Adams, Cooperative Atom-light Interaction in a Blockaded Rydberg Ensemble, Phys. Rev. Lett. 105, 193603 (2010).

[39] I. Lesanovsky, Many-Body Spin Interactions and the Ground State of a Dense Rydberg Lattice Gas, Phys. Rev. Lett. 106, 025301 (2011).

[40] H. Weimer and H.P. Büchler, Two-Stage Melting in Systems of Strongly Interacting Rydberg Atoms, Phys. Rev. Lett. 105, 230403 (2010).

[41] T. Pohl, E. Demler, and M. D. Lukin, Dynamical Crystallization in the Dipole Blockade of Ultracold Atoms, Phys. Rev. Lett. 104, 043002 (2010).

[42] J. Schachenmayer, I. Lesanovsky, A. Micheli, and A. J. Daley, Dynamical crystal creation with polar molecules or Rydberg atoms in optical lattices, New J. Phys. 12, 103044 (2010).

[43] G. Pupillo, A. Micheli, M. Boninsegni, I. Lesanovsky, and P. Zoller, Strongly Correlated Gases of Rydberg-Dressed Atoms: Quantum and Classical Dynamics, Phys. Rev. Lett. 104, 223002 (2010).

[44] H. Weimer, R. Löw, T. Pfau, and H. P. Büchler, Quantum Critical Behavior in Strongly Interacting Rydberg Gases, Phys. Rev. Lett. 101, 250601 (2008).

[45] T. E. Lee, H. Häffner, and M. C. Cross, Collective Quantum Jumps of Rydberg Atoms, Phys. Rev. Lett. 108, 023602 (2012).

[46] M. Marcuzzi, E. Levi, S. Diehl, J. P. Garrahan, and I. Lesanovsky, Universal Nonequilibrium Properties of Dissipative Rydberg Gases, Phys. Rev. Lett. 113, 210401 (2014).

[47] E. A. Goldschmidt, T. Boulier, R. C. Brown, S. B. Koller, J. T. Young, A. V. Gorshkov, S. L. Rolston, and J. V. Porto, Anomalous broadening in driven dissipative Rydberg systems, Phys. Rev. Lett. 116, 113001 (2016). 